\title{
Comparison Between Single Dose Paravertebral Block and Thoracic Epidural Block for Breast Surgeries : A Prospective Randomized Study
}

\author{
Authors: Pai V.K, Singh A.P., Singh D.K., Dhar M.
}

Institute of Medical Sciences, Banaras Hindu University, Dept of Anaesthesiology, Varanasi, India

\section{INTRODUCTION}

Both paravertebral block (PVB) and thoracic epidural block (TEB) are established techniques for postoperative pain relief in thoracic and breast surgeries. Breast surgeries are associated with postoperative pain, nausea and vomiting. With medical advances and rise in number of ambulatory surgeries there is a growing interest towards regional anaesthesia for breast surgeries.

\section{AIMS AND OBJECTIVES}

To study and compare

* The anaesthetic and hemodynamic effects of paravertebral block \& thoracic epidural block intraoperatively.

* The side effects and patient satisfaction in breast surgery patients using the two techniques.

\section{MATERIALS AND METHODS}

After ethical committee approval, 80 patients scheduled for unilateral simple mastectomy were randomized to receive either TEB or PVB. Written informed consent was taken from all the participants.

Patients with severe cardiovascular, respiratory, endocrine disease, bleeding disorders, allergy to any of the study drug, kyphoscoliosis, body mass index $>35$ were excluded from the study.

\section{Procedure for thoracic paravertebral block}

A point $2.5 \mathrm{~cm}$ lateral to the upper border of spinous processes of the $\mathrm{T} 2$ vertebra was marked, area was prepared, painted and draped and space infiltrated with $2 \mathrm{~mL}$ of $1 \%$ lignocaine.

A $20 \mathrm{G}$ needle was introduced perpendicular to the skin in all planes to touch the transverse process of the lower vertebra. After identification of the transverse process, the needle was walked off the superior surface of the transverse process and slowly advanced $1-1.5 \mathrm{~cm}$ until a loss of resistance to air was obtained with a 5-mL glass syringe.

$10-15 \mathrm{ml}$ of $0.5 \%$ Bupivaciane solution was then injected after repeated negative aspiration for blood or cerebrospinal fluid, in graded doses.

\section{Procedure for Epidural:}

After identification of level, painting and draping, skin was infiltrated with local anaesthetic using 25 -gauge 1.5 inch needle.

Epidural needle with stylette was inserted through same skin puncture using midline approach and the glass syringe attached to the hub of the needle. After confirming LOR to air, $10-15 \mathrm{ml}$ of $0.5 \%$ Bupivacaine was injected in graded doses.

\section{The following measurements were recorded:} drug.

Performance time: Time between needle insertion and injection of

Onset of action: Time between injection of drug and unresponsiveness to unilateral needle prick in atleast three segments.

Duration of surgery: Time between incision and application of adhesive bandage and closure of wound.

Intraoperatively, patients received an IV infusion of Propofol (30-70 $\mathrm{mcg} / \mathrm{kg} / \mathrm{min}$ ). Intermittent doses of Fentanyl $25 \mathrm{mcg}$ and Propofol $10 \mathrm{mg}$ were given for supplemental sedation if Ramsey sedation score of $>2$.

Patient satisfaction was recorded four hours after surgery as excellent, very good, good and poor.

\section{STATISTICAL ANALYSIS}

Statistical analysis was done by computer statistical software system SPSS ver 16. and results are presented in tabulated manner. Comparison between groups were performed by using unpaired ' $\mathrm{t}$ ' test or Chi-square test, as appropriate. The results are expressed in mean $\pm \mathrm{SD}$ and number $(\%)$.

\section{RESULTS}

\begin{tabular}{|l|c|c|c|}
\hline & Group TEB (n=30) & Group PVB (n=30) & P value \\
\hline Age & $38.67 \pm 17.93$ & $39.33 \pm 18.93$ & 0.412 \\
\hline BMI & $21.69 \pm 2.57$ & $22.27 \pm 1.97$ & 0.138 \\
\hline Heart rate & $96.33 \pm 10.63$ & $92.33 \pm 9.77$ & 0.449 \\
\hline Mean arterial pressure & $81.76 \pm 6.70$ & $83.37 \pm 7.38$ & 0.385 \\
\hline
\end{tabular}

$(\mathrm{n}=30$ in each group), all the baseline characteristics and vital parameters of the patients in two groups were comparable.

Table 2. Mean blood pressure.

Table 2. Mean blood pressure.
\begin{tabular}{|c|c|c|c|c|c|}
\hline \multirow{2}{*}{ Time } & \multicolumn{2}{|c|}{ Group TEB $(\mathbf{n}=\mathbf{3 0})$} & \multicolumn{2}{c|}{ Group PVB $(\mathbf{n}=\mathbf{3 0}$} & \multirow{2}{*}{ P value } \\
\cline { 2 - 5 } & Range & Mean \pm SD & Range & Mean $\pm \mathbf{S D}$ & \\
\hline $\mathbf{0} \mathbf{~ m i n}$ & $87.15 \pm 8.62$ & $71-99$ & $87.15 \pm 8.62$ & $75-101$ & 0.385 \\
\hline $\mathbf{1 0} \mathbf{~ m i n}$ & $72.91 \pm 10.40$ & $54-91$ & $85.16 \pm 10.37$ & $73-98$ & $0.0008^{*}$ \\
\hline $\mathbf{2 0} \mathbf{~ i n}$ & $73 \pm 11.07$ & $55-92$ & $85.15 \pm 9.42$ & $73-98$ & $0.0014^{*}$ \\
\hline $\mathbf{3 0} \mathbf{~ m i n}$ & $73.43 \pm 10.11$ & $56-92$ & $85.95 \pm 9.02$ & $72-99$ & 0.118 \\
\hline $\mathbf{4 0} \mathbf{~ m i n}$ & $78.93 \pm 9.56$ & $68-92$ & $84.63 \pm 7.69$ & $70-98$ & 0.219 \\
\hline $\mathbf{5 0} \mathbf{~ m i n}$ & $79.73 \pm 10.51$ & $67-93$ & $84.77 \pm 6.98$ & $70-95$ & 0.258 \\
\hline $\mathbf{1}$ hour & $82.5 \pm 10.12$ & $69-95$ & $84.65 \pm 8.62$ & $69-98$ & 0.323 \\
\hline $\mathbf{1}$ hour po & $84.27 \pm 9.54$ & $69-97$ & $86.15 \pm 9.62$ & $71-97$ & 0.345 \\
\hline
\end{tabular}

Blood Pressure measured in $\mathrm{mm}$ of $\mathrm{Hg}$ showed significant fall in epidural group and statistically significant difference was found between Group E and Group P at 10 min and 20 min.

Table 3. Observations

\begin{tabular}{|c|c|c|c|c|c|}
\hline \multirow{2}{*}{ Time } & \multicolumn{2}{|c|}{ Group TEB $(n=30)$} & \multicolumn{2}{|c|}{ Group PVB $(n=30)$} & \multirow{2}{*}{$\begin{array}{c}P \\
\text { VALUE }\end{array}$} \\
\hline & Range & Mean \pm SD & Range & Mean \pm SD & \\
\hline Performance Time (in min) & $7.47 \pm 1.98$ & $5-10$ & $6.8 \pm 1.74$ & $4-10$ & 0.288 \\
\hline Induction Time (in $\mathrm{min}$ ) & $17.68 \pm 1.98$ & $13-23$ & $17.57 \pm 1.98$ & $14-21$ & 0.101 \\
\hline Duration of Surgery (in $\mathrm{min}$ ) & $67.17 \pm 31.18$ & $38-127$ & $69.33 \pm 31.90$ & $25-120$ & 0.392 \\
\hline VAS score PO & $0.37 \pm 0.43$ & $0-1$ & $0.53 \pm 0.56$ & $0-2$ & 0.323 \\
\hline VAS score $1 \mathrm{hr}$ PO & $1.1 \pm 1.44$ & $0-4$ & $1.03 \pm 1.17$ & $0-4$ & 0.304 \\
\hline
\end{tabular}

Table 4. Side effects

\begin{tabular}{|l|c|c|c|c|}
\hline \multirow{2}{*}{ Side effects } & \multicolumn{2}{|c|}{ Group TEB $(\mathbf{n = 3 0})$} & \multicolumn{2}{c|}{ Group PVB $(\mathbf{n = 3 0})$} \\
\cline { 2 - 5 } & Number & Percent & Number & Percent \\
\hline Hypotension & 3 & $10 \%$ & 0 & 0 \\
\hline Nausea vomiting & 6 & $20 \%$ & 2 & $7 \%$ \\
\hline Others & 0 & 0 & 0 & 0 \\
\hline
\end{tabular}

Table 5. Patient satisfaction

\begin{tabular}{|l|c|c|c|}
\hline & Group TEB (n=30) & Group PVB $(\mathbf{n = 3 0})$ & P value \\
\hline Excellent & $14(46.67 \%)$ & $17(56.66 \%)$ & 0.446 \\
\hline Very good & $10(33.33 \%)$ & $7(23.33 \%)$ & 0.483 \\
\hline Good & $6(20 \%)$ & $6(20 \%)$ & 0.893 \\
\hline Poor & 0 & 0 & 0.887 \\
\hline
\end{tabular}

\section{CONCLUSION}

Single injection PVB and TEB had similar quality of surgical anaesthesia, post-operative pain relief and patient satisfaction however PVB had better hemodynamic stability than TEB in patients undergoing breast surgeries. Further data and studies will be required to confirm this preliminary study.

\section{REFERENCES}

1. Shikha Soni, Ankit Soni, Manjula Bapugol, Sadik Mohammed, Rakesh Karnawat, K L. Tulsiani. Comparison of thoracic epidural block vs paravertebral block in patients under going breast surgery. Indian Journal of Clinical Anaesthesia, January - March 2015;2(1):48-56.

2. Coveney E, Weltz CR, Greengrass R, Iglehart JD, Leight GS, Steele SM, et al. Use of paravertebral block anesthesia in the surgical management of breast cancer: Experience in 156 cases. Ann Surg 1998;227:496-501.

3. Lynch EP, Welch KJ, Carabuena JM, Eberlein TJ. Thoracic epidural anesthesia improves outcome after breast surgery. Ann Surg 1995;222:663-9. 